\title{
CHARACTER VALUES AT INVOLUTIONS
}

\author{
P. X. GALLAGHER
}

(Communicated by Ronald M. Solomon)

\begin{abstract}
If $\chi_{1}, \chi_{2}, \chi_{3}$ are irreducible characters of a finite group $G$ satisfying $\int_{G} \chi_{1} \chi_{2} \chi_{3} \neq 0$ and $\sigma$ is an involution in $G$, then the proportions of -1 's among the eigenvalues of the corresponding representations at $\sigma$ are the sides of a triangle on a sphere of circumference 2 .
\end{abstract}

For an involution $\sigma$ of a finite group $G$ and an irreducible complex representation $R$ of $G$, denote by $q$ the proportion of -1 's among the eigenvalues of $R(\sigma)$. The objects of this note are to prove the bounds

$$
\frac{1}{h} \leq q \leq 1-\frac{1}{h}, \quad \text { unless } q=0 \text { or } 1,
$$

where $h$ is the index of the centralizer $C$ of $\sigma$, and then, refining (1), to prove "spherical triangle" inequalities

$$
q_{i}+q_{j}+q_{k} \leq 2, \quad q_{i} \leq q_{j}+q_{k}, \quad \text { etc. },
$$

for each triple $R_{i}, R_{j}, R_{k}$ for which

$$
R_{i} \otimes R_{j} \otimes R_{k} \mid G
$$

contains the principal representation. Finally, we show the equivalence of:

(a) $q=1-\frac{1}{h}$ (or $\frac{1}{h}$ )

(b) $R$ is induced by the unique irreducible representation $S$ in $R \mid C$ whose restriction to $\langle\sigma\rangle$ is a multiple of the principal (nonprincipal) representation; and

(c) there is equality in one of the first two (last two) inequalities in (2) with $R_{i}=R$ and all $R_{j}, R_{k}$ satisfying (3).

Proof of (1). Let $R$ represent $G$ on a space $V$ of dimension $f$ with character $\chi$. Let $T$ be one of the two irreducible representations of $\langle\sigma\rangle$, and let $V_{T}$ be the $T$-isotypic subspace of $V$. If $V_{T} \neq 0$, then $V=\sum_{\tau \in G} \tau V_{T}$ since $R$ is irreducible. Since $\tau V_{T}=V_{T^{\tau}}$ depends only on $\tau \bmod C$, it follows that

$$
V=\sum_{\tau \bmod C} \tau V_{T},
$$

Received by the editors May 5, 1992.

1991 Mathematics Subject Classification. Primary $20 \mathrm{C} 15$.

Key words and phrases. Finite groups, character values, involutions.

C)1994 American Mathematical Society 
so $f \leq$ he where $e$ is the dimension of $V_{T}$. Since $e=(1-q) f$ or $q f$ according as $T$ is principal or not, the inequalities in (1) follow from hypotheses $V_{T} \neq 0$ in the two cases.

Proof. (a) $\Leftrightarrow(\mathrm{b})$. By the above argument, $q=1-\frac{1}{h}$ (or $\frac{1}{h}$ ) is equivalent to a direct sum in (4) with $T$ principal (or nonprincipal). Let $S$ be the representation of $C$ on $V_{T}$. A direct sum in (4) means that $R$ is induced by $S$, which must then be the unique irreducible representation in $R \mid C$ whose restriction to $\langle\sigma\rangle$ is a multiple of $T$. Conversely, if $R$ is induced by such an $S$, then

$$
\chi(\sigma)= \pm e \mp(f-e)=\mp f\left(1-\frac{2}{h}\right),
$$

giving

$$
q=\frac{1}{2}\left(1-\frac{\chi(\sigma)}{f}\right)=1-\frac{1}{h}\left(\text { or } \frac{1}{h}\right)
$$

for $T$ principal (or nonprincipal).

Proof. (3) $\Rightarrow(2)$. Put

$$
P=\int_{G} R_{i} \otimes R_{j} \otimes R_{k}, \quad c=c_{i j k}=\int_{G} \chi_{i} \chi_{j} \chi_{k},
$$

and for irreducible representations $T_{i}, T_{j}, T_{k}$ of $\langle\sigma\rangle$ put

$$
P^{\prime}=\int_{\langle\sigma\rangle} \int_{\langle\sigma\rangle} \int_{\langle\sigma\rangle}\left(\psi_{i} \otimes \psi_{j} \otimes \psi_{k}\right)\left(R_{i} \otimes R_{j} \otimes R_{k}\right),
$$

where the $\psi$ 's are the characters of the $T$ 's. Relative to a $G \times G \times G$ invariant positive definite Hermitian form on $V_{i} \otimes V_{j} \otimes V_{k}, P$ and $P^{\prime}$ are selfadjoint idempotents, the orthogonal projections of $V_{i} \otimes V_{j} \otimes V_{k}$ onto the subspace of $G$-invariants and the subspace $\left(V_{i}\right)_{T_{i}} \otimes\left(V_{j}\right)_{T_{j}} \otimes\left(V_{k}\right)_{T_{k}}$ respectively. It follows that

$$
\operatorname{tr}\left(P^{\prime} P\right)=\operatorname{tr}\left(\left(P^{\prime} P\right)\left(P^{\prime} P\right)^{*}\right) \geq 0 .
$$

From $(5)$ and $(6), \operatorname{tr}\left(P^{\prime} P\right)$ is

$$
\int_{\langle\sigma\rangle} \int_{\langle\sigma\rangle} \int_{\langle\sigma\rangle} \psi_{i}\left(\sigma_{i}\right) \psi_{j}\left(\sigma_{j}\right) \psi_{k}\left(\sigma_{k}\right) \int_{G} \chi_{i}\left(\sigma_{i} \tau\right) \chi_{j}\left(\sigma_{j} \tau\right) \chi_{k}\left(\sigma_{k} \tau\right) .
$$

According as $\sigma_{i}, \sigma_{j}, \sigma_{k}$ are the same $(1,1,1$ or $\sigma, \sigma, \sigma)$ or not, e.g., with $\sigma_{i} \neq \sigma_{j}=\sigma_{k}$, the inner integral is $c$ or, e.g., $c \chi_{i}(\sigma) / f_{i}$. Choosing $T_{i}, T_{j}, T_{k}$ all principal or only $T_{i}$ principal gives

$$
\operatorname{tr}\left(P^{\prime} P\right)=\frac{c}{4}\left(1+\frac{\chi_{i}(\sigma)}{f_{i}} \pm \frac{\chi_{j}(\sigma)}{f_{j}} \pm \frac{\chi_{k}(\sigma)}{f_{k}}\right) .
$$

Assuming $c \neq 0$, which is (3), the first two inequalities in (2) follow from (7) and (8) using $\chi(\sigma) / f=1-2 q$. The last two inequalities in (2) follow similarly.

Proof. (3) $\Rightarrow(2)$ implies (1) and (a) $\Leftrightarrow(c)$. From the orthogonality relations it 
follows that

$$
\begin{aligned}
\sum_{j, k} c_{i j k} f_{j} f_{k} & =|G| f_{i}, \\
\sum_{j, k} c_{i j k} \chi_{j}^{2}(\sigma) / f_{j} & =|C| f_{i}, \\
\sum_{j, k} c_{i j k} \chi_{j}(\sigma) \chi_{k}(\sigma) & =|C| \chi_{i}(\sigma) .
\end{aligned}
$$

In terms of $a_{i j k}=c_{i j k} f_{j} f_{k} / f_{i}$ and $x_{i}=\chi_{i}(\sigma) / f_{i}$, these equations are

$$
\sum_{j, k} a_{i j k}=|G|, \quad \sum_{j, k} a_{i j k} x_{j}^{2}=|C|, \quad \sum_{j, k} a_{i j k} x_{j} x_{k}=|C| x_{i} .
$$

Using $a_{i j k} \geq 0$ and $1 \pm x_{i} \geq\left|x_{j} \pm x_{k}\right|$ for $a_{i j k} \neq 0$, it follows that

$$
\left(1 \pm x_{i}\right)^{2}|G| \geq \sum_{j, k} a_{i j k}\left(x_{j} \pm x_{k}\right)^{2}=2\left(1 \pm x_{i}\right)|C|
$$

which implies $1 \pm x_{i} \geq \frac{2}{h}$ for $x_{i} \neq \mp 1$, which is (1) again. It also follows that (a) is equivalent to $1 \pm x_{i}=\left|x_{j} \pm x_{k}\right|$ for all $j, k$ with $a_{i j k} \neq 0$, i.e., for + to equality in the first or second of the inequalities in (2) for all $j, k$ with $c_{i j k} \neq 0$, and for - to equality in the third or fourth of the inequalities in (2) for all $j, k$ with $c_{i j k} \neq 0$.

Department of Mathematics, Columbia University, New York, New York, 10027 